\title{
Parkinson's Symptoms and Caregiver Burden and Mental Health: A Cross-Cultural Mediational Model
}

\author{
Erin R. Smith $\mathbb{D}^{1}$, Paul B. Perrin $\mathbb{D}^{1,2}$ Carmen M. Tyler, ${ }^{1}$ Sarah K. Lageman $\left(\mathbb{D},{ }^{3}\right.$ \\ and Teresita Villaseñor ${ }^{4,5}$ \\ ${ }^{1}$ Department of Psychology, Virginia Commonwealth University, USA \\ ${ }^{2}$ Department of Physical Medicine and Rehabilitation, Virginia Commonwealth University, USA \\ ${ }^{3}$ Department of Neurology, Parkinson's and Movement Disorders Center, Virginia Commonwealth University, USA \\ ${ }^{4}$ Hospital Civil Fray Antonio Alcalde, Mexico \\ ${ }^{5}$ Department of Neurosciences, University of Guadalajara, Mexico
}

Correspondence should be addressed to Paul B. Perrin; pperrin@vcu.edu

Received 3 August 2019; Revised 15 October 2019; Accepted 6 November 2019; Published 1 December 2019

Academic Editor: Marjan Jahanshahi

Copyright (c) 2019 Erin R. Smith et al. This is an open access article distributed under the Creative Commons Attribution License, which permits unrestricted use, distribution, and reproduction in any medium, provided the original work is properly cited.

Informal caregivers are critical in the care of individuals with Parkinson's disease (PD) and spend substantial time providing care, which may be associated with negative caregiver outcomes such as burden and mental health issues. Although research in the United States and Europe has generally supported these relations, there is very limited research on PD caregiving in Latin America. The current study examined the following connections in a sample of PD caregivers from the United States $(N=105)$ and Mexico ( $N=148$ ): (a) PD-related impairments (motor and nonmotor symptoms) and caregiver burden, (b) caregiver burden and caregiver mental health, and (c) PD-related impairments and mental health through caregiver burden. Study results uncovered significant relations among PD-related impairments, caregiver burden, and caregiver mental health. Further, caregiver burden fully mediated the relation between PD-related impairments and caregiver mental health at both study sites. Findings highlight a number of important intervention targets for caregivers and families, including caregiver burden and mental health.

\section{Introduction}

Parkinson's disease (PD) is a progressive neurodegenerative condition that leads to physical disability [1] and cognitive impairment [2] over time, both of which may limit an individual's independent functioning. PD is the second most common progressive neurodegenerative disease in the United States [3], affecting approximately $1 \%$ of individuals over the age of 60 [4]. Recent estimates suggest that by 2030, there will be approximately 1.2 million individuals in the United States living with PD [5]. Similarly, due to its aging population [6], rates of PD are likely to increase rapidly in Latin America, with estimates suggesting the prevalence of PD will double in Mexico within 20 years [7] since rates of PD increase with age [8].
There are an estimated 129,124 individuals living with PD in Central Latin America, 30,717 in Andean Latin America, and 131,748 in Tropical Latin America [9]. However, despite the high prevalence and increasing rates of $\mathrm{PD}$ in Latin America, very little is known about either PD patient or caregiver experiences in the region and how experiences may differ from those in the United States.

$\mathrm{PD}$ is characterized by its classic motor symptoms, including akinesia (loss or impaired voluntary movement), bradykinesia (slowness of movement), resting tremor (shaking while in a relaxed state), and postural instability [10]. Individuals may present with additional motor symptoms, including gait problems [11] as well as reduced facial expression [12]. By the time an individual is 10 years postdiagnosis, the individual 
will present with a number of nonmotor symptoms [13] that can include depression, cognitive impairment, apathy, anxiety, sleep disruption, dementia, and psychosis [14].

Given the progressive nature of the disease, impairment increases over time, leading most individuals living with PD to require the assistance of a caregiver. This care is often provided by an informal caregiver, an individual who does not receive financial compensation for caregiving and is usually a family member $[15,16]$. Informal caregivers support the individual with PD by performing a number of physical, social, and emotional tasks, which may include assisting with personal care and activities of daily living (e.g., bathing, feeding, and administering medications), transportation and mobility assistance (e.g., getting in and out of bed), providing social and emotional support, and financially supporting the individual [17-19]. Providing care may come at a significant cost to the caregiver, such as giving up a career, leisure activities, or social activities to take care of the individual with PD [17], and negative consequences for the caregiver may include having a lower quality of life than the general population $[20,21]$. These factors contribute to the caregiver becoming what has been described as an "invisible patient," and the toll of caregiving on a caregiver's life may diminish the overall effectiveness of the care they provide [14].

These substantial life changes may result in caregiver burden, a multidimensional construct that has been operationalized in a number of ways. For example, Zarit et al. [22] describe caregiver burden as encompassing the adverse effects caregiving may have on an individual's emotional, financial, social, physical, and spiritual function which may engender feelings of discomfort due to the demands, time constraints, duties, and difficulties of providing care. Successive researchers have built upon this definition by adding the internal conflict that caregivers may experience when they are unable to fulfill their personal needs due to caregiving [23], as well as including the reactions informal caregivers may have to the emotional, social, physical, and financial difficulties that result from providing care [24]. Caregiver burden is critical to examine, as it may lead to a reduced sense of well-being and burnout [25].

One of the first studies to examine motor symptoms and caregiver burden was conducted by Carter et al. [26], who found that individuals who were rated by clinicians to be in the later Hoehn and Yahr [27] stages of PD had spousal caregivers with higher caregiver strain than those in early stages. Other studies generally support the relationship between motor impairments and burden. For example, Martínez-Martín et al. [21] examined Hoehn and Yahr [27] staging and caregiver burden in a sample of Spanish caregivers and found that PD severity was a primary predictor of caregiver burden. Increasing attention has also been given to the nonmotor symptoms of PD [14], which may be difficult for caregivers to address. Anywhere from 25 to $40 \%$ of individuals living with PD eventually develop dementia [28] and may experience related deficits in attention, memory, and executive functioning [14]. Leroi et al. [29] found that caregiver burden was significantly higher in caregivers who cared for an individual with PD who had dementia than those without cognitive impairment or with only mild cognitive impairment, suggesting that dementia is independently associated with increased caregiver burden.

The mental health of PD caregivers has been found to be lower than that of the general population [30], suggesting that caregiving may take a toll on an individual's mental health. Depression and anxiety specifically may be critical to examine as they can elicit cognitive biases that engender greater feelings of caregiver burden [14, 31]. At least one study has suggested that the link between depression and caregiver burden may be particularly critical in PD compared to other neurological diseases (e.g., Alzheimer's disease [32]).

To date, very little research has examined PD in regions outside of North America and Europe. The majority of research conducted outside of these regions has focused on data derived from medical records or drug consumption data [33]. This is problematic for low-to-middle-income countries, as these estimates inherently exclude individuals who are unable to obtain medical care or prescription drugs to treat PD [34]. Further, these studies have also not considered the unique culturally determined treatment practices and varying access to care for PD throughout the world [35]. As such, there is a critical need to examine PD caregiving in diverse regions of the world, such as Latin America. Given the rapidly aging population in both the United States [5] and Mexico [7], rates of PD are likely to rise in both countries, suggesting that the number of individuals providing informal care will also increase. As a result, the current study examined the following connections in two samples of PD caregivers from the United States and Mexico: (a) PD-related impairments (motor and nonmotor symptoms) and caregiver burden, (b) caregiver burden and caregiver mental health, and (c) PD-related impairments and mental health through caregiver burden. The current study presents the results of a secondary analysis of data from another manuscript comparing PD caregiver and patient characteristics in the United States and Mexico [36]. This other manuscript found that caregivers in the United States site were older than those in Mexico, spent fewer hours per week providing care, provided care to an older individual with $\mathrm{PD}$, and had provided care for a longer duration of time, although there were no differences in the number of individuals who assisted the caregiver in providing care. In the current study, it was hypothesized that caregiver burden would mediate the association between PD-related impairments and mental health across both sites.

\section{Method}

2.1. Participants. This study used cross-sectional data that were collected from PD caregivers from the Hospital Civil Fray Antonio Alcalde of the University of Guadalajara in Guadalajara, Mexico, and the Parkinson's and Movement Disorders Center (PMDC) at Virginia Commonwealth University in Henrico, Virginia. Both centers offer interdisciplinary models of health care for patients as well as services for caregivers. The Hospital Civil Fray Antonio Alcalde also offers education programs and emotional support groups led by psychologists and lodging and food 
TABLE 1: Characteristics of PD caregivers and patients.

\begin{tabular}{lcc}
\hline Demographic variable & Value \\
\hline & United States & Mexico \\
Age & & \\
$\quad$ Caregiver (years, mean (SD)) & $68.73(8.36)$ & $53.66(14.96)$ \\
Patient (years, mean (SD)) & $71.61(8.13)$ & $65.68(10.78)$ \\
Sex (\%) & & \\
Caregiver & & \\
$\quad$ Female & $68.6 \%$ & $76.4 \%$ \\
$\quad$ Male & $31.4 \%$ & $23.6 \%$ \\
Patient & & \\
$\quad$ Female & $35.2 \%$ & $48.0 \%$ \\
$\quad$ Male & $64.8 \%$ & $52.0 \%$ \\
Months providing care (mean (SD)) & $46.78(81.33)$ & $52.38(49.22)$ \\
Relationship to individual with PD (\%) & & \\
Parent & $3.8 \%$ & $34.5 \%$ \\
Aunt/uncle & $1.0 \%$ & $1.4 \%$ \\
Spouse/romantic partner & $93.3 \%$ & $51.4 \%$ \\
Sibling & $0 \%$ & $7.4 \%$ \\
Child & $0 \%$ & $0 \%$ \\
Friend & $1.9 \%$ & $.7 \%$ \\
Cousin & $0 \%$ & $.7 \%$ \\
Other & $0 \%$ & $4.1 \%$ \\
\hline
\end{tabular}

support for caregivers. To be eligible for this study, participants had to have met the following inclusion criteria: (a) identify as a caregiver of an individual diagnosed with PD, (b) be at least 18 years of age, and (c) be fluent in either English (for the United States site) or Spanish (for the Mexico site). Please see Table 1 for PD caregiver and patient characteristics.

In the United States sample $(N=105)$, the majority of caregivers self-identified as women (68.6\%). Caregivers had a mean age of $68.73(\mathrm{SD}=8.36)$ and were predominantly spouses or partners (93.3\%) of individuals with PD. On average, caregivers had provided care for 49.05 months and 60.43 hours per week. The majority of individuals selfidentified as White/European American (92.4\%), followed by Asian/Asian American/Pacific Islander (2.9\%), Black/African American (non-Latino; 2.9\%), multiracial/multiethnic $(1.0 \%)$, or other identity (1.0\%). Of the sample, $25.7 \%$ had a high school education or equivalent, 2-year technical degree (11.4\%), 4-year college degree (33.3\%), master's degree $(21.9 \%)$, or doctorate degree $(7.6 \%)$.

In the Mexico sample $(N=148)$, the majority of caregivers self-identified as women (76.4\%) and had a mean age of $53.66(\mathrm{SD}=14.96)$. Over half of the caregivers were spouses or partners of the individual living with $\mathrm{PD}$ (51.4\%). On average, caregivers provided care for 52.38 $(\mathrm{SD}=49.22)$ months and $107.39(\mathrm{SD}=61.34)$ hours per week. Of the sample, $4.7 \%$ had no formal education, $58.1 \%$ had an elementary school education, 5.4\% had a high school education or equivalent, $13.5 \%$ had a 2 -year technical degree, $16.2 \%$ had a 4 -year college degree, and $2.0 \%$ had a master's degree.

\subsection{Measures}

2.2.1. PD Impairments. PD-related impairments were assessed using the Movement Disorder Society-Unified Parkinson's Disease Rating Scale (MDS-UPDRS; [37]). Participants were instructed to answer this questionnaire based on their observations and experiences with the individual living with PD. Participants responded to two subscales within the questionnaire: Part I (nonmotor aspects of experiences of daily living) and Part II (motor aspects of experiences of daily living). Participants respond to each item on a Likert-type scale that ranges from 0 (normal: no problems present) to 4 (severe: problems are present and preclude the patient's ability to carry out normal activities or social interactions or to maintain previous standards in personal or family life). In the present study, total scores for each subscale were created by summing scores for each item within the subscale. The nonmotor experiences of the daily living subscale has acceptable internal consistency $(\alpha=.79)$, and the motor experiences of daily living has good internal consistency $(\alpha=.90$; [37]). Additionally, for symptom-based exploratory analyses, scores were calculated for the following subdomains on the MDS-UPDRS: cognitive/mental health (items 1.1-1.6), sleep and fatigue (items 1.7, 1.8, and 1.13), bowel and bladder (items 1.10 and 1.11), pain (item 1.9), lightheadedness (item 1.12), activities of daily living (items 2.5-2.8), physical impairment (items 2.9-2.13 and 1.12), and speech/oral (items 2.1-2.4). The Movement Disorder Society (MDS) has also translated and validated the scale in Spanish, with the nonmotor experiences of daily living demonstrating acceptable internal consistency $(\alpha=.79)$ and the motor experiences of daily living demonstrating good internal consistency $(\alpha=.92 ;[38])$. In the current study, the nonmotor subscale demonstrated good internal consistency in the Mexico $(\alpha=.80)$ and the United States sample $(\alpha=.85)$. The motor subscale also demonstrated good internal consistency in the Mexico $(\alpha=.88)$ and the United States sample $(\alpha=.90)$. No specific instructions were given regarding responding when the patient was "on" or "off" medication status.

2.2.2. Caregiver Burden. The short version of the Zarit Burden Inventory [39] was used to assess caregiver burden. Participants responded to the 12-item version of the ZBI on a Likert-type scale from 0 (never) to 4 (nearly always), where higher scores indicate higher levels of caregiver burden. The full version of the ZBI has previously been validated in caregivers of individuals living with Parkinson's disease [21]. The ZBI has also been validated in Spanish-speaking individuals and demonstrated good internal consistency $(\alpha=.92 ;[40])$. In the current study, the measure demonstrated good internal consistency in the Mexico $(\alpha=.86)$ and United States samples $(\alpha=.91)$.

2.2.3. Mental Health. To compute the mental health variable, $z$-scores from the Patient Health Questionnaire-9 (PHQ-9; [41]), assessing for depressive symptomatology, and the Generalized Anxiety Disorder-7 (GAD-7; [42]), assessing for anxiety, were averaged. 
(1) Depression. Participants responded to the 9-item PHQ-9 [41] on a Likert-type scale from 0 (not at all) to 3 (nearly every day), where higher scores correspond to greater depressive symptomatology. The PHQ-9 has previously been validated in Spanish-speaking individuals and demonstrated good internal consistency $(\alpha=.92$; [43-45]). In the current study, the PHQ-9 had good internal consistency in the Mexico $(\alpha=.81)$ and United States samples $(\alpha=.82)$.

(2) Anxiety. Participants responded to the 7-item GAD-7 [42] on a Likert-type scale from 0 (not at all) to 3 (nearly every day). Participants' scores may range from 0 to 21, with higher scores indicating greater levels of anxiety. The GAD-7 has previously been translated and validated in Spanish and demonstrated excellent internal consistency $(\alpha=.92$; [46]). In the current study, the GAD-7 demonstrated good internal consistency in the Mexico $(\alpha=.88)$ and United States samples $(\alpha=.90)$.

2.3. Procedure. The protocol for the current study was reviewed and approved by the Institutional Review Boards at both data collection sites, Virginia Commonwealth University and the Hospital Civil Fray Antonio Alcalde. Participants were recruited by research assistants at both sites using written and verbal advertisements, predominantly from waiting rooms but also via clinician referral after medical appointments. Email advertisements were also sent to a listserv at the PMDC at Virginia Commonwealth University. At each data collection location, interested individuals were provided with information on the study in the respective clinic and provided informed consent prior to enrolling in the study. Participants were screened for eligibility, and if eligible, completed all study measures. The protocol was orally administered at the Hospital Civil Fray Antonio Alcalde site to collect demographic and questionnaire data in order to account for higher rates of illiteracy than at the United States site. The oral interview took approximately an hour. Participants from the PMDC completed all survey measures independently using pencil and paper. Completion of study measures took participants approximately the same amount of time.

2.4. Data Analyses. Four mediational models were created to determine if caregiver burden mediates the relationship between PD-related impairments and caregiver mental health. The first model used motor impairments as a predictor and a second model used nonmotor impairments as a predictor. Each of these models was run separately by site for a total of four mediational models using the PROCESS macro [47]. This macro utilizes the Preacher and Hayes $[48,49]$ asymptotic bootstrapping approach. In this approach, a large number of samples are taken from the data, and by sampling with replacement, the indirect effect from each sample is calculated. A sample of 5,000 bootstrap samples was taken as recommended by Preacher and Hayes $[48,49]$. The indirect effect estimate was calculated by taking the mean of all of the indirect effects across the bootstrap samples. In this approach, statistical significance is determined by creating a confidence interval surrounding the indirect effect. The analysis used a $95 \%$ confidence interval with an $\alpha$ level of .05. The null hypothesis is rejected (i.e., no indirect effect) if the obtained confidence interval does not contain zero. All predictor variables (motor and nonmotor impairments, caregiver burden) were meancentered prior to running analyses. Finally, an exploratory step-wise multiple regression was run predicting caregiver burden, wherein the first step included important demographic variables as predictors and the second step included symptom clusters of PD (cognitive/mental health, sleep and fatigue, bowel and bladder, pain, lightheadedness, activities of daily living, physical impairment, and speech/oral).

\section{Results}

3.1. Motor Impairments. Please see Table 2 for a summary of PD motor impairments. For the Mexico site, the overall model was significant, $F(2,145)=5.54, p<.001$, and $R^{2}=.07$. The direct path from motor symptoms to caregiver burden was statistically significant $(b=.32, p<.001)$, as was the path from caregiver burden to caregiver mental health $(b=.06, p=.0016)$. The motor impairment model demonstrated a mean bootstrap estimate of the indirect effect of $b=.02$. The obtained confidence interval did not contain zero $(.01, .04)$, suggesting that caregiver burden mediates the association between motor impairments and caregiver mental health among caregivers from the Mexico site. Overall, this suggests that greater motor impairments predicted greater caregiver burden, which predicted greater mental health deficits among caregivers. The direct effect between motor impairments and mental health was $b=-.01$ and was not significant $(p=.677)$, suggesting that caregiver burden fully mediated the association between motor symptoms and caregiver mental health.

Similar results were obtained for the United States site. The overall model was significant, $F(2,102)=26.48$, $p<.001$, and $R^{2}=.34$. The direct path from motor symptoms to caregiver burden was statistically significant $(b=.44$, $p<.001)$, as was the path from caregiver burden to caregiver mental health $(b=.14, p<.001)$. The motor impairment model demonstrated a mean bootstrap estimate of the indirect effect of $b=.06$. The obtained confidence interval did not contain zero $(.04, .09)$, suggesting that caregiver burden mediated the association between motor impairments and caregiver mental health among caregivers from the United States site. Given that the direct effect between motor impairments and mental health was $b=-.03$ and was not significant $(p=.097)$, this suggests that caregiver burden fully mediated the association between motor impairments and caregiver mental health.

3.2. Nonmotor Impairments. Please see Table 2 for a summary of PD nonmotor impairments. For the Mexico site, the overall model was significant, $F(2,145)=6.46, p=.002$, and $R^{2}=.08$. The direct path from nonmotor symptoms to caregiver burden was statistically significant $(b=.48$, $p<.001)$ and the direct path from caregiver burden to caregiver mental health was significant $(b=.05, p=.026)$. The nonmotor impairment model demonstrated a mean 
TABLE 2: Summary of symptoms reported by caregivers $(N=253)$. \begin{tabular}{lll}
\hline Symptom domain & Symptom endorsed & $\begin{array}{l}\text { \% endorsing symptom } \\
\text { United States Mexico }\end{array}$
\end{tabular}

\begin{tabular}{|c|c|c|c|}
\hline & Cognitive impairm & & \\
\hline & Normal & $26.7 \%$ & $39.2 \%$ \\
\hline & Slight & $27.6 \%$ & $29.1 \%$ \\
\hline & Mild & $17.1 \%$ & $15.5 \%$ \\
\hline & Moderate & $23.8 \%$ & $11.5 \%$ \\
\hline & Severe & $4.8 \%$ & $4.7 \%$ \\
\hline & $\begin{array}{c}\text { Hallucinations an } \\
\text { psychosis }\end{array}$ & & \\
\hline & Normal & $78.1 \%$ & $82.4 \%$ \\
\hline & Slight & $12.4 \%$ & $9.5 \%$ \\
\hline & Mild & $5.7 \%$ & $4.1 \%$ \\
\hline & Moderate & $2.9 \%$ & $2.7 \%$ \\
\hline & Severe & $1.0 \%$ & $1.4 \%$ \\
\hline & Depressed mooc & & \\
\hline & Normal & $37.1 \%$ & $33.1 \%$ \\
\hline & Slight & $41.0 \%$ & $24.3 \%$ \\
\hline & Mild & $11.4 \%$ & $17.6 \%$ \\
\hline & Moderate & $8.6 \%$ & $20.3 \%$ \\
\hline & Severe & $1.9 \%$ & $4.7 \%$ \\
\hline & Anxious mood & & \\
\hline & Normal & $36.2 \%$ & $31.3 \%$ \\
\hline & Slight & $38.1 \%$ & $31.8 \%$ \\
\hline Nonmotor & Mild & $17.1 \%$ & $16.2 \%$ \\
\hline & Moderate & $6.7 \%$ & $18.2 \%$ \\
\hline & Severe & $1.9 \%$ & $2.7 \%$ \\
\hline & Apathy & & \\
\hline & Normal & $39.0 \%$ & $56.1 \%$ \\
\hline & Slight & $35.2 \%$ & $17.6 \%$ \\
\hline & Mild & $18.1 \%$ & $13.5 \%$ \\
\hline & Moderate & $3.8 \%$ & $10.5 \%$ \\
\hline & Severe & $3.8 \%$ & $2.7 \%$ \\
\hline & $\begin{array}{l}\text { Dopamine } \\
\text { dysregulation } \\
\text { syndrome }\end{array}$ & & \\
\hline & Normal & $68.6 \%$ & $79.1 \%$ \\
\hline & Slight & $16.2 \%$ & $8.1 \%$ \\
\hline & Mild & $11.4 \%$ & $5.4 \%$ \\
\hline & Moderate & $3.8 \%$ & $4.7 \%$ \\
\hline & Severe & $0.0 \%$ & $2.7 \%$ \\
\hline & Sleep problems & & \\
\hline & Normal & $19.0 \%$ & $33.1 \%$ \\
\hline & Slight & $22.9 \%$ & $20.9 \%$ \\
\hline & Mild & $27.6 \%$ & $16.9 \%$ \\
\hline & Moderate & $23.8 \%$ & $19.6 \%$ \\
\hline & Severe & $5.7 \%$ & $9.5 \%$ \\
\hline
\end{tabular}

TABle 2: Continued.

Symptom domain Symptom endorsed

$\%$ endorsing symptom United States Mexico

Daytime sleepiness

$\begin{array}{ccc}\text { Normal } & 14.3 \% & 29.1 \% \\ \text { Slight } & 24.8 \% & 25.0 \% \\ \text { Mild } & 51.5 \% & 25.0 \% \\ \text { Moderate } & 6.7 \% & 16.9 \% \\ \text { Severe } & 2.9 \% & 4.1 \%\end{array}$

Pain and other

sensations

Normal

$21.9 \% \quad 30.4 \%$

Slight

$36.2 \%$

$31.3 \%$

Mild

$15.3 \%$

$21.6 \%$

Moderate

$16.2 \% \quad 14.2 \%$

Severe

$10.5 \%$

$2.7 \%$

Urinary problems

Normal

$38.1 \% \quad 54.1 \%$

Slight

$26.7 \%$

$23.0 \%$

Mild

$15.2 \%$

$10.1 \%$

Moderate

$13.3 \%$

$9.5 \%$

Severe

$6.7 \%$

$3.4 \%$

Constipation

Normal

Slight

$33.3 \%$

$43.2 \%$

Mild

$37.1 \%$

$23.0 \%$

Moderate

$16.2 \%$

$18.9 \%$

Severe

$13.3 \%$

$12.8 \%$

Lightheadedness on

$0.0 \%$

$2.0 \%$

standing

Normal

$52.4 \%$

$65.5 \%$

Slight

$27.6 \%$

$19.6 \%$

Mild

$10.5 \%$

$5.4 \%$

Moderate

$9.5 \%$

$8.1 \%$

Severe

$0.0 \%$

$1.4 \%$

Fatigue

Normal

$18.1 \% \quad 34.5 \%$

Slight

$41.0 \%$

$27.7 \%$

Mild

$25.7 \%$

$18.2 \%$

Moderate

$10.5 \%$

$14.2 \%$

Severe

$4.8 \%$

$5.4 \%$

Speech

$\begin{array}{ccc}\text { Normal } & 24.8 \% & 38.5 \% \\ \text { Slight } & 19.0 \% & 25.7 \% \\ \text { Mild } & 29.5 \% & 16.9 \% \\ \text { Moderate } & 23.8 \% & 12.8 \% \\ \text { Severe } & 2.9 \% & 6.1 \%\end{array}$


TABLE 2: Continued.

\begin{tabular}{|c|c|c|c|}
\hline Symptom domain & Symptom endorsed & \multicolumn{2}{|c|}{ \% endorsing symptom } \\
\hline \multicolumn{4}{|c|}{ Saliva/drooling } \\
\hline & Normal & $57.1 \%$ & $60.8 \%$ \\
\hline & Slight & $12.4 \%$ & $13.5 \%$ \\
\hline & Mild & $11.4 \%$ & $16.2 \%$ \\
\hline & Moderate & $9.5 \%$ & $8.1 \%$ \\
\hline & Severe & $9.5 \%$ & $1.4 \%$ \\
\hline \multicolumn{4}{|c|}{$\begin{array}{l}\text { Chewing and } \\
\text { swallowing }\end{array}$} \\
\hline & Normal & $55.2 \%$ & $72.3 \%$ \\
\hline & Slight & $36.2 \%$ & $10.8 \%$ \\
\hline & Mild & $6.7 \%$ & $10.8 \%$ \\
\hline & Moderate & $1.0 \%$ & $4.7 \%$ \\
\hline & Severe & $0.0 \%$ & $1.4 \%$ \\
\hline \multicolumn{4}{|c|}{ Eating tasks } \\
\hline & Normal & $45.7 \%$ & $43.2 \%$ \\
\hline & Slight & $28.6 \%$ & $33.1 \%$ \\
\hline & Mild & $21.9 \%$ & $16.2 \%$ \\
\hline & Moderate & $2.9 \%$ & $6.8 \%$ \\
\hline & Severe & $1.0 \%$ & $0.7 \%$ \\
\hline \multicolumn{4}{|c|}{ Dressing } \\
\hline & Normal & $28.6 \%$ & $22.3 \%$ \\
\hline & Slight & $21.0 \%$ & $52.0 \%$ \\
\hline & Mild & $37.1 \%$ & $15.5 \%$ \\
\hline & Moderate & $7.6 \%$ & $7.4 \%$ \\
\hline & Severe & $5.7 \%$ & $2.7 \%$ \\
\hline \multicolumn{4}{|c|}{ Hygiene } \\
\hline & Normal & $42.9 \%$ & $27.7 \%$ \\
\hline & Slight & $31.4 \%$ & $49.3 \%$ \\
\hline & Mild & $17.1 \%$ & $15.5 \%$ \\
\hline & Moderate & $4.8 \%$ & $4.1 \%$ \\
\hline & Severe & $3.8 \%$ & $3.4 \%$ \\
\hline \multicolumn{4}{|c|}{ Handwriting } \\
\hline & Normal & $22.9 \%$ & $31.8 \%$ \\
\hline & Slight & $25.7 \%$ & $33.8 \%$ \\
\hline & Mild & $22.9 \%$ & $14.9 \%$ \\
\hline & Moderate & $21.0 \%$ & $11.5 \%$ \\
\hline & Severe & $7.6 \%$ & $8.1 \%$ \\
\hline \multicolumn{4}{|c|}{$\begin{array}{l}\text { Hobbies and other } \\
\text { activities }\end{array}$} \\
\hline & Normal & $27.6 \%$ & $27.7 \%$ \\
\hline & Slight & $20.0 \%$ & $21.6 \%$ \\
\hline & Mild & $25.7 \%$ & $15.5 \%$ \\
\hline & Moderate & $17.1 \%$ & $23.0 \%$ \\
\hline & Severe & $9.5 \%$ & $12.2 \%$ \\
\hline \multicolumn{4}{|c|}{ Turning in bed } \\
\hline & Normal & $38.1 \%$ & $37.2 \%$ \\
\hline & Slight & $41.9 \%$ & $35.8 \%$ \\
\hline & Mild & $12.4 \%$ & $16.2 \%$ \\
\hline
\end{tabular}

TABle 2: Continued.

\begin{tabular}{lll}
\hline Symptom domain $\quad$ Symptom endorsed & $\begin{array}{l}\text { \% endorsing symptom } \\
\text { United States Mexico }\end{array}$ \\
\hline
\end{tabular}

$\begin{array}{ccc}\text { Moderate } & 5.7 \% & 7.4 \% \\ \text { Severe } & 1.9 \% & 3.4 \% \\ \text { Tremor } & & \\ \text { Normal } & 26.7 \% & 18.9 \% \\ \text { Slight } & 46.7 \% & 44.6 \% \\ \text { Mild } & 18.1 \% & 18.2 \% \\ \text { Moderate } & 6.7 \% & 14.2 \% \\ \text { Severe } & 1.9 \% & 4.1 \%\end{array}$

Getting out of bed,

car, or deep chair

$\begin{array}{ccc}\text { Normal } & 14.3 \% & 31.1 \% \\ \text { Slight } & 34.3 \% & 28.4 \% \\ \text { Mild } & 29.5 \% & 25.0 \% \\ \text { Moderate } & 15.2 \% & 10.8 \% \\ \text { Severe } & 6.7 \% & 4.7 \%\end{array}$

Walking and

balancing

Normal $\quad 14.3 \% \quad 23.6 \%$

Slight $\quad 42.0 \% \quad 39.9 \%$

Mild $\quad 14.3 \% \quad 16.2 \%$

Moderate $\quad 24.8 \% \quad 15.5 \%$

Severe $\quad 4.8 \% \quad 4.7 \%$

Freezing

Normal $\quad 52.4 \% \quad 60.8 \%$

Slight $\quad 20.0 \% \quad 18.9 \%$

Mild $\quad 6.7 \% \quad 6.8 \%$

Moderate $\quad 15.2 \% \quad 8.8 \%$

Severe $\quad 5.7 \% \quad 4.7 \%$

bootstrap estimate of the indirect effect of $b=.02$. The obtained confidence interval did not contain zero $(.0036, .04)$, suggesting that caregiver burden mediates the association between nonmotor impairments and caregiver mental health among caregivers from the Mexico site. Similar to the prior model, these results suggest that greater nonmotor impairments predicted greater caregiver burden, which predicted greater mental health deficits among caregivers. The direct effect between nonmotor symptoms and caregiver mental health was $b=.03$ and was not significant $(p=.172)$, suggesting that caregiver burden fully mediated the association between nonmotor impairments and caregiver mental health among caregivers from the Mexico site.

Again, similar results were obtained for the United States site. The overall model was significant, $F(2,102)=25.63$, $p<.001$, and $R^{2}=.33$. The direct path from nonmotor symptoms to caregiver burden was significant $(b=.65$, $p<.001)$ and the direct path from caregiver burden to caregiver mental health was significant $(b=.14, p<.001)$. The model demonstrated a mean bootstrap estimate of the indirect effect of $b=.09$. The obtained confidence interval 
did not contain zero $(.05, .14)$, suggesting that caregiver burden mediated the association between nonmotor impairments and caregiver mental health among caregivers from the United States site. The direct effect between nonmotor impairments and mental health was $b=-.03$ and was not significant $(p=.203)$, suggesting that caregiver burden fully mediated the association between nonmotor impairments and caregiver mental health.

3.3. Exploratory Analysis. In the step-wise regression predicting burden, the first step with demographic predictors (caregiver age, caregiver gender, hours per week providing care, months providing care, caregiver education, and number of individuals who assisted the caregiver in providing care) was statistically significant, $F(6,240)=2.65, p=.017$, and $R^{2}=.06$. Within the overall model, the only unique predictor was caregiver education $(\beta=.17, p=.014)$. In the second step, PD symptom clusters were added as predictors, resulting in an overall statistically significant model, $F(8,232)=14.70, p<.001$, and $R^{2}=.38$. Within the overall model, the only unique predictor was caregiver cognitive/mental health symptoms $(\beta=.35, p<.001)$.

\section{Discussion}

The current study examined PD caregivers in Henrico, Virginia, and Guadalajara, Mexico, and the relations among PD-related impairments, caregiver burden, and caregiver mental health. Results from both the motor and nonmotor impairments models suggest that caregiver burden fully mediates the association between PD-related impairments and caregiver mental health in both caregivers from the United States and from Mexico. Generally, prior research has supported the links among PD-related impairments and caregiver burden [14], caregiver burden and mental health [31], and PD-related impairments and mental health $[20,50]$. To date, this is the first evidence that caregiver burden fully mediates the relations between both PD-related motor and nonmotor impairments and mental health. One possible explanation for these results is that as PD-related impairments become more severe, levels of caregiver burden increase, which in turn may lead to poorer mental health.

PD-related impairments, particularly nonmotor symptoms, were associated with deleterious outcomes for caregivers in the current study. Further, the exploratory multiple regression suggested that cognitive/mental health symptoms had the biggest impact on caregiver burden. These findings are similar to those of Schrag et al. [51], who found that PD caregiver burden was particularly related to nonmotor symptoms including depression, hallucinations, and confusion. The current findings in light of the previous research suggest that it may be important for health care providers of caregivers to be mindful of nonmotor PD-related impairments and how they may affect the psychosocial functioning and well-being of the caregiver. Interventions that target PD-related impairments may also be useful in reducing caregiver burden and have already received some support in the literature. For example, interventions that promote independence and functioning among individuals with $\mathrm{PD}$ may be associated with reduced caregiver burden. Recently, exercise interventions for individuals with $\mathrm{PD}$ have received attention. Oguh et al. [52] found that individuals with PD who exercised more than 150 minutes a week had better quality of life, physical function, and reduced disease progression, as well as less caregiver burden among their caregivers than those who were not regular exercisers.

Targeting nonmotor symptoms may also serve to reduce deleterious outcomes for caregivers, such as caregiver burden and mental health issues. For example, previous studies have shown that addressing dementia with cognitive enhancers has been associated with reduced caregiver burden [53, 54]. Prior research has also demonstrated that tailored cognitive behavioral therapy for individuals with PD with anxiety is associated with reduced caregiver burden [55]. Overall, these studies suggest that there is benefit in considering PD-related impairments for caregivers, particularly as it may relate to burden. It is important to note that these studies were not conducted in Latin America or with diverse samples. As such, future research should seek to determine if these interventions are also effective among individuals with $\mathrm{PD}$ and caregivers in other geographic regions and among racially/ethnically diverse samples.

The current study suggests that reducing caregiver burden is an important target for intervention as it channels directly into mental health. To date, there have been a number of interventions that target burden among caregivers of individuals with $\mathrm{PD}$. One educational intervention that addressed the scheduling of pleasant activities, communication, reducing burden, and managing stress was shown to significantly reduce burden from baseline [56]. Further, interventions emphasizing education and fellowship with other caregivers have also been reported in the literature and have received qualitative support for reducing caregiver burden $[18,57,58]$. However, again, none of these interventions were conducted in Latin America and did not consist of diverse samples (in terms of race/ethnicity, languages spoken, etc.). Therefore, it is unclear if these interventions would be effective in this geographical region. As such, another critical target for intervention is the development and evaluation of interventions that may be culturally adapted for caregivers living in Latin America.

One such cultural consideration would be the influence of gender-role expectations as they contribute to caregiver burden. Links between female PD caregivers and increased anxiety have been found in some studies [20] while others have found no differences in mental health outcomes by sex [59]. However, there is evidence that Mexican gender-role expectations influence the amount and types (e.g., instrumental and emotional) of caregiving done by each sex; women take on markedly more caregiving duties than men, which may provide insight into potential lower mental health ratings [60]. Another cultural consideration is that the only demographic predictor of caregiver burden was education, such that higher education was associated with greater caregiver burden. This could be because caregivers with a higher educational level may have additional work-related duties that contribute unique stressors on top of heavy caregiving responsibilities. It would be important to consider whether 
educational attainment plays a differential role in caregiving in the U.S. versus Mexico, as U.S. caregivers have higher educational attainment. Future cross-cultural studies should attempt to tease out the contributions of gender roles and education in PD caregiver burden.

Given the demonstrated associations between mental health and deleterious outcomes for caregivers (e.g., burden), caregiver mental health may be an important intervention target. To date, at least one cognitive behavioral intervention has been shown to reduce caregiver burden among caregivers who report emotional distress. Secker and Brown [61] found that 12 to 14 sessions of cognitive behavioral therapy focused on relaxation, sleep hygiene, accessing support, and challenging negative beliefs delivered by a clinical psychologist reduced burden six months postintervention compared to the control group. Given the lack of mental health interventions for PD caregivers in Latin America, interventions that are culturally tailored for this population may serve to address the mental health needs of PD caregivers in a culturally sensitive manner.

\subsection{Limitations and Future Research}

4.1.1. Methodological Weaknesses in Data Collection. The present study is limited in that it only recruited from two outpatient clinics: a specialty clinic in Henrico, Virginia, and a clinic in Guadalajara, Mexico. As such, the current study likely did not capture caregivers of individuals in the later stages of PD when individuals are likely to be institutionalized [62]. Given evidence demonstrating caregiver burden is highest in stage IV immediately prior to institutionalization at stage $\mathrm{V}$ [62], the relationships identified in the current study among outpatient caregivers may not generalize to all PD caregivers.

The data in the current study were collected using a slightly different methodology at the Mexico site and the United States site. At the United States site, caregivers completed the study measures independently using paper and pencil. In contrast, at the Mexico site, researchers used oral interviews to collect data from participants (in order to account for potential problems with illiteracy), which may have influenced the responses of participants. In addition, the study measures utilized in the current study (apart from the MDS-UPDRS) were validated for self-report and not for oral interviews. Therefore, it is possible that caregivers from the Mexico site responded differently from caregivers from the United States site.

Another limitation is that the data in this study were collected exclusively from caregivers. Therefore, the data in the current study represent their perceptions of PD-related impairments and caregiver burden and mental health. Future studies should also aim to use more objective measures such as patient medical records to assess PD-related impairments. It may be particularly helpful to collect objective information on disease stage, which has been associated with deleterious outcomes for caregivers such as burden [21] and which unfortunately was not collected in the current study. Furthermore, collecting data regarding whether or not the patient is currently taking PD medication may be informative, as dopamine agonists and L-dopa (which are often used to address PD symptomatology) may have a number of side effects. The unintended effects of these pharmaceutical agents may include nausea, impulsive behaviors (including impulse control disorders), dopamine dysregulation syndrome, and psychosis [63], which may result in negative consequences for the caregiver such as lower quality of life $[19,20]$.

4.1.2. Cross-Sectional Methodology. Since the current study was cross-sectional in nature, causal inferences cannot be made. Future research should utilize cross-lagged panel designs or other longitudinal methods to infer whether the relations identified in the current study may be causal in nature. Further, it is possible that some of the relationships are reciprocal. For example, it is possible that mental health problems also influence levels of caregiver burden.

4.1.3. Generalizability. Given that the samples in the current study came from two clinics, it is possible that the experiences of these caregivers may not generalize to the population of caregivers of individuals living with $\mathrm{PD}$ in the United States and Mexico. For example, caregivers at the United States site were recruited from a PD specialty clinic in a suburban area, suggesting that the individuals they care for are at least receiving some health care. Similarly, participants from the Mexico site were recruited from a large, urban hospital, also suggesting they have at least some access to health care. The experiences of caregivers without health care, such as those in rural areas, may differ greatly from caregivers who do have health care.

\section{Conclusions}

The current study examined associations among PD-related impairments, caregiver burden, and caregiver mental health among caregivers of individuals with $\mathrm{PD}$ residing in the United States and Mexico. Findings from the current study as well as prior literature highlight the importance of targeting critical caregiver outcomes such as caregiver burden and caregiver mental health.

\section{Data Availability}

The data used to support the findings of this study are available from the corresponding author upon request.

\section{Conflicts of Interest}

The authors report no conflicts of interest.

\section{References}

[1] L. M. Shulman, M. Armstrong, T. Ellis et al., "Disability rating scales in Parkinson's disease: critique and recommendations," Movement Disorders, vol. 31, no. 10, pp. 1455-1465, 2016.

[2] M. Petrou, B. A. Dwamena, B. R. Foerster et al., “Amyloid deposition in Parkinson's disease and cognitive impairment: a systematic review," Movement Disorders, vol. 30, no. 7, pp. $928-935,2015$. 
[3] L. M. L. de Lau and M. M. B. Breteler, "Epidemiology of Parkinson's disease," The Lancet Neurology, vol. 5, no. 6, pp. 525-535, 2006.

[4] R. L. Nussbaum and C. E. Ellis, “Alzheimer's disease and Parkinson's disease," The New England Journal of Medicine, vol. 348, no. 14, pp. 1356-1364, 2003.

[5] on behalf of the Parkinson's Foundation P4 Group, C. Marras, J. C. Beck et al., "Prevalence of Parkinson's disease across North America," Npj Parkinson's Disease, vol. 4, no. 1, 2018.

[6] R. Wong and A. Palloni, "Aging in Mexico and Latin America," in International Handbook of Population Aging, Springer, Dordrecht, 2009.

[7] L. Cantu-Martinez, J. Santos-Guzman, I. Estrada-Bellman, R. Mora-Villalvazo, E. Salinas-Lezama, and H. Martinez, "Economic burden of Parkinson's disease in a population of Monterrey, Mexico," Neurology, vol. 82, 10 Supplement, 2014Retrieved from http://n.neurology.org/content/82/10_ Supplement/P4.054.abstract.

[8] J. V. Hindle, "Ageing, neurodegeneration and Parkinson's disease," Age and Ageing, vol. 39, no. 2, pp. 156-161, 2010.

[9] E. R. Dorsey, A. Elbaz, E. Nichols et al., "Global, regional, and national burden of Parkinson's disease, 1990-2016: a systematic analysis for the Global Burden of Disease Study 2016," The Lancet Neurology, vol. 17, no. 11, pp. 939-953, 2018.

[10] A. S. Mandir and C. Vaughan, "Pathophysiology of Parkinson's disease," International Review of Psychiatry, vol. 12, no. 4, pp. 270-280, 2000.

[11] E. B. Forsaa, J. P. Larsen, T. Wentzel-Larsen, and G. Alves, "A 12-year population-based study of freezing of gait in Parkinson's disease," Parkinsonism \& Related Disorders, vol. 21, no. 3, pp. 254-258, 2015.

[12] L. Ricciardi, M. Bologna, F. Morgante et al., "Reduced facial expressiveness in Parkinson's disease: A pure motor disorder?," Journal of the Neurological Sciences, vol. 358, no. 1-2, pp. 125-130, 2015.

[13] W. Poewe, "The natural history of Parkinson's disease," Journal of Neurology, vol. 253, no. S7, pp. vii2-vii6, 2006.

[14] P. E. Mosley, R. Moodie, and N. Dissanayaka, "Caregiver burden in Parkinson disease: a critical review of recent literature," Journal of Geriatric Psychiatry and Neurology, vol. 30, no. 5, pp. 235-252, 2017.

[15] R. Buchanan, D. Radin, B. J. Chakravorty, and T. Tyry, "Perceptions of informal care givers: health and support services provided to people with multiple sclerosis," Disability and Rehabilitation, vol. 32, no. 6, pp. 500-510, 2010.

[16] C. Zucchella, M. Bartolo, C. Pasotti, L. Chiapella, and E. Sinforiani, "Caregiver burden and coping in early-stage Alzheimer disease," Alzheimer Disease \& Associated Disorders, vol. 26, no. 1, pp. 55-60, 2012.

[17] R. Bhimani, "Understanding the Burden on Caregivers of People with Parkinson's: A Scoping Review of the Literature," Rehabilitation Research and Practice, vol. 2014, 8 pages, 2014.

[18] D. McLaughlin, F. Hasson, W. G. Kernohan et al., "Living and coping with Parkinson's disease: perceptions of informal carers," Palliative Medicine, vol. 25, no. 2, pp. 177-182, 2011.

[19] National Alliance for Caregiving, "Caregiving in the U.S.," 2015, Retrieved from https://www.caregiving.org/pdf/ research/Caregiving_in_the_US_2009_full_report.pdf.

[20] P. Martinez-Martin, S. Arroyo, J. M. Rojo-Abuin et al., "Burden, perceived health status, and mood among care- givers of Parkinson's disease patients," Movement Disorders, vol. 23, no. 12, pp. 1673-1680, 2008.

[21] P. Martínez-Martín, M. J. Forjaz, B. Frades-Payo et al., "Caregiver burden in Parkinson's disease," Movement Disorders, vol. 22, no. 7, pp. 924-931, 2007.

[22] S. H. Zarit, P. A. Todd, and J. M. Zarit, "Subjective burdens of husbands and wives as caregivers: a longitudinal study," Alzheimer Disease \& Associated Disorders, vol. 1, no. 2, pp. 109110, 1987.

[23] V. Braithwaite, "Understanding stress in informal Caregiving," Research on Aging, vol. 18, no. 2, pp. 139-174, 1996.

[24] R. T. Kasuya, P. Polgar-Bailey, and R. Takeuchi, "Caregiver burden and burnout. A guide for primary care physicians," Postgraduate Medicine, vol. 108, no. 7, pp. 119-123, 2000.

[25] M. Thornton and S. S. Travis, "Analysis of the reliability of the modified caregiver strain index," The Journals of Gerontology Series B: Psychological Sciences and Social Sciences, vol. 58, no. 2, pp. S127-S132, 2003.

[26] J. H. Carter, B. J. Stewart, P. G. Archbold et al., "Living with a person who has Parkinson's disease: the spouse's perspective by stage of disease. Parkinson's Study Group," Movement Disorders, vol. 13, no. 1, pp. 20-28, 1998.

[27] M. M. Hoehn and M. D. Yahr, "Parkinsonism: onset, progression, and mortality," Neurology, vol. 17, no. 5, pp. 427-442, 1967.

[28] A. Elbaz, J. H. Bower, B. J. Peterson et al., "Survival study of Parkinson disease in Olmsted County, Minnesota," Archives of Neurology, vol. 60, no. 1, pp. 91-96, 2003.

[29] I. Leroi, K. McDonald, H. Pantula, and V. Harbishettar, "Cognitive impairment in Parkinson disease: impact on quality of life, disability, and caregiver burden," Journal of Geriatric Psychiatry and Neurology, vol. 25, no. 4, pp. 208-214, 2012.

[30] M. Peters, R. Fitzpatrick, H. Doll, D. Playford, and C. Jenkinson, "Does self-reported well-being of patients with Parkinson's disease influence caregiver strain and quality of life?," Parkinsonism \& Related Disorders, vol. 17, no. 5, pp. 348-352, 2011.

[31] D. Grün, V. Pieri, M. Vaillant, and N. J. Diederich, "Contributory factors to caregiver burden in Parkinson disease," Journal of the American Medical Directors Association, vol. 17, no. 7, pp. 626-632, 2016.

[32] H. Shin, J. Youn, J. S. Kim, J.-Y. Lee, and J. W. Cho, “Caregiver burden in Parkinson disease with dementia compared to Alzheimer disease in Korea," Journal of Geriatric Psychiatry and Neurology, vol. 25, no. 4, pp. 222-226, 2012.

[33] T. Pringsheim, N. Jette, A. Frolkis, and T. D. L. Steeves, "The prevalence of Parkinson's disease: a systematic review and meta-analysis," Movement Disorders, vol. 29, no. 13, pp. 1583-1590, 2014.

[34] M. C. de Rijk, C. Tzourio, M. M. Breteler et al., "Prevalence of parkinsonism and Parkinson's disease in Europe: the EUROPARKINSON Collaborative Study. European Community Concerted Action on the Epidemiology of Parkinson's disease," Journal of Neurology, Neurosurgery, and Psychiatry, vol. 62, no. 1, pp. 10-15, 1997.

[35] A. Chiò, C. Magnani, and D. Schiffer, "Prevalence of Parkinson's disease in northwestern Italy: comparison of tracer methodology and clinical ascertainment of cases," Movement Disorders, vol. 13, no. 3, pp. 400-405, 1998.

[36] E. R. Smith, P. B. Perrin, C. M. Tyler, T. Villaseñor, and S. K. Lageman, (in preparation) Cross-cultural differences in caregiving and burden between the United States and Mexico.. 
[37] C. G. Goetz, B. C. Tilley, S. R. Shaftman et al., "Movement Disorder Society-sponsored revision of the Unified Parkinson's Disease Rating Scale (MDS-UPDRS): scale presentation and clinimetric testing results," Movement Disorders, vol. 23, no. 15, pp. 2129-2170, 2008.

[38] P. Martinez-Martin, C. Rodriguez-Blazquez, M. AlvarezSanchez et al., "Expanded and independent validation of the Movement Disorder Society-Unified Parkinson's Disease Rating Scale (MDS-UPDRS)," Journal of Neurology, vol. 260, no. 1, pp. 228-236, 2013.

[39] M. Bédard, D. W. Molloy, L. Squire, S. Dubois, J. A. Lever, and M. O'Donnell, "The Zarit Burden Interview: a new short version and screening version," The Gerontologist, vol. 41, no. 5, pp. 652-657, 2001.

[40] M. Marín, "Adaptación para nuestro medio de la Escala de Sobrecarga del Cuidador de Zarit," Revista Multidisciplinar de Gerontología, vol. 6, no. 4, 1996.

[41] K. Kroenke, R. L. Spitzer, and J. B. Williams, "The PHQ-9: validity of a brief depression severity measure," Journal of General Internal Medicine, vol. 16, no. 9, pp. 606-613, 2001.

[42] R. L. Spitzer, K. Kroenke, J. B. W. Williams, and B. Löwe, “A brief measure for assessing generalized anxiety disorder: the GAD-7," Archives of Internal Medicine, vol. 166, no. 10, pp. 1092-1097, 2006.

[43] C. Diez-Quevedo, T. Rangil, L. Sanchez-Planell, K. Kroenke, and R. L. Spitzer, "Validation and utility of the patient health questionnaire in diagnosing mental disorders in 1003 general hospital Spanish inpatients," Psychosomatic Medicine, vol. 63, no. 4, pp. 679-686, 2001.

[44] W. Donlan and J. Lee, "Screening for depression among indigenous Mexican migrant farmworkers using the Patient Health Questionnaire-9," Psychological Reports, vol. 106, no. 2, pp. 419-432, 2010.

[45] L. Wulsin, E. Somoza, and J. Heck, "The feasibility of using the Spanish PHQ-9 to screen for depression in primary care in Honduras," Primary Care Companion to The Journal of Clinical Psychiatry, vol. 04, no. 05, pp. 191-195, 2002.

[46] J. García-Campayo, E. Zamorano, M. A. Ruiz et al., "Cultural adaptation into Spanish of the Generalized Anxiety Disorder-7 (GAD-7) scale as a screening tool," Health and Quality of Life Outcomes, vol. 8, p. 8, 2010.

[47] A. F. Hayes, Introduction to mediation, moderation, and conditional process analysis: A regression-based approach, Guilford Publications, 2nd ed. edition, 2017.

[48] K. J. Preacher and A. F. Hayes, "SPSS and SAS procedures for estimating indirect effects in simple mediation models," Behavior Research Methods, Instruments, \& Computers, vol. 36, no. 4, pp. 717-731, 2004.

[49] K. J. Preacher and A. F. Hayes, "Asymptotic and resampling strategies for assessing and comparing indirect effects in multiple mediator models," Behavior Research Methods, vol. 40, no. 3, pp. 879-891, 2008.

[50] H. H. Fernandez, R. E. J. Tabamo, R. D. Raymund, and J. H. Friedman, "Predictors of depressive symptoms among spouse caregivers in Parkinson's disease," Movement Disorders, vol. 16, no. 6, pp. 1123-1125, 2001.

[51] A. Schrag, A. Hovris, D. Morley, N. Quinn, and M. Jahanshahi, "Caregiver-burden in parkinson's disease is closely associated with psychiatric symptoms, falls, and disability," Parkinsonism \& Related Disorders, vol. 12, no. 1, pp. 35-41, 2006.
[52] O. Oguh, A. Eisenstein, M. Kwasny, and T. Simuni, "Back to the basics: regular exercise matters in Parkinson's disease: results from the National Parkinson Foundation QII Registry study," Parkinsonism \& Related Disorders, vol. 20, no. 11, pp. 1221-1225, 2014.

[53] I. V. Litvinenko, M. M. Odinak, V. I. Mogil'naya, and A. Y. Emelin, "Efficacy and safety of galantamine (reminyl) for dementia in patients with Parkinson's disease (an open controlled trial)," Neuroscience and Behavioral Physiology, vol. 38, no. 9, pp. 937-945, 2008.

[54] P. J. Reading, A. K. Luce, and I. G. McKeith, "Rivastigmine in the treatment of parkinsonian psychosis and cognitive impairment: preliminary findings from an open trial," Movement Disorders, vol. 16, no. 6, pp. 1171-1174, 2001.

[55] N. N. W. Dissanayaka, D. Pye, L. K. Mitchell et al., "Cognitive behavior therapy for anxiety in Parkinson's disease: outcomes for patients and caregivers," Clinical Gerontologist, vol. 40, no. 3, pp. 159-171, 2017.

[56] G. Simons, S. B. N. Thompson, and M. C. Smith Pasqualini, "An innovative education programme for people with Parkinson's disease and their carers," Parkinsonism \& Related Disorders, vol. 12, no. 8, pp. 478-485, 2006.

[57] K. P. Roland, M. E. Jenkins, and A. M. Johnson, “An exploration of the burden experienced by spousal caregivers of individuals with Parkinson's disease," Movement Disorders, vol. 25, no. 2, pp. 189-193, 2010.

[58] A. Schrag, D. Morley, N. Quinn, and M. Jahanshahi, "Impact of Parkinson's disease on patients' adolescent and adult children," Parkinsonism \& Related Disorders, vol. 10, no. 7, pp. 391-397, 2004.

[59] K. Hooker, M. Manoogian-O’Dell, D. J. Monahan, L. D. Frazier, and K. Shifren, "Does type of disease matter? Gender differences among Alzheimer's and Parkinson's disease spouse caregivers," The Gerontologist, vol. 40, no. 5, pp. 568-573, 2000.

[60] P. B. Perrin, I. Panyavin, A. Morlett Paredes et al., "A disproportionate burden of care: gender differences in mental health, health-related quality of life, and social support in Mexican multiple sclerosis caregivers," Behavioural Neurology, vol. 2015, Article ID 283958, 9 pages, 2015.

[61] D. L. Secker and R. G. Brown, "Cognitive behavioural therapy (CBT) for carers of patients with Parkinson's disease: a preliminary randomised controlled trial," Journal of Neurology, Neurosurgery \& Psychiatry, vol. 76, no. 4, pp. 491-497, 2005.

[62] Deloitte Access Economics, "Living with Parkinson's disease: an updated economic analysis 2014," 2015, Retrieved March 17, 2018, from Parkinson's Queensland website: http://www.parkinsons-qld.org.au/living-with-parkinsonsdisease-an-updated-economic-analysis-2014/.

[63] B. S. Connolly and A. E. Lang, "Pharmacological treatment of parkinson disease," JAMA, vol. 311, no. 16, pp. 1670-1683, 2014. 


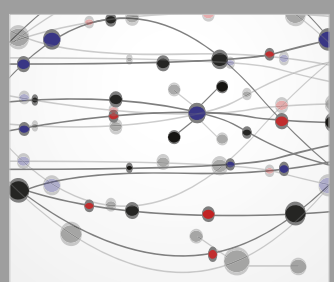

The Scientific World Journal
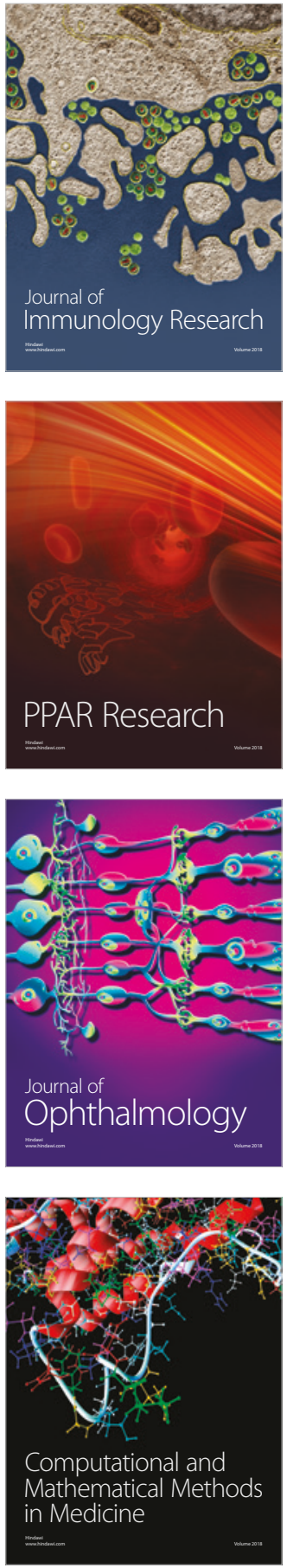

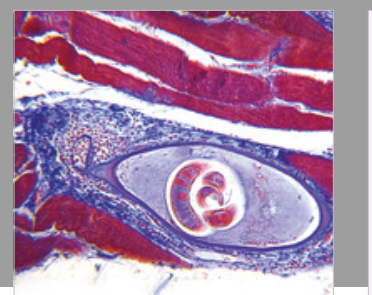

Gastroenterology Research and Practice

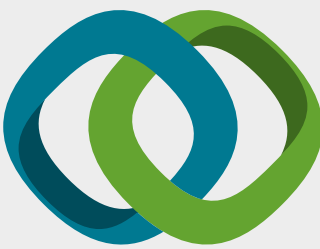

\section{Hindawi}

Submit your manuscripts at

www.hindawi.com
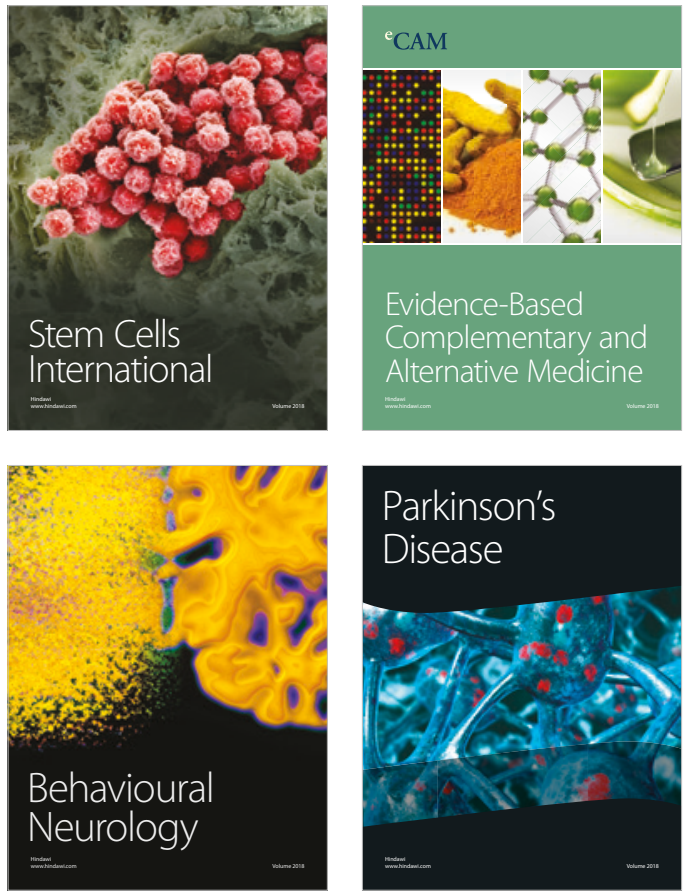

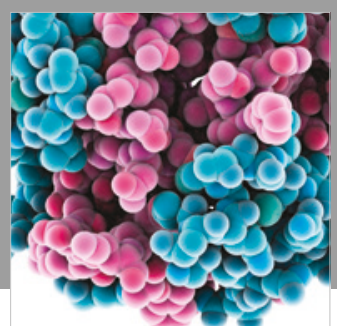

ournal of

Diabetes Research

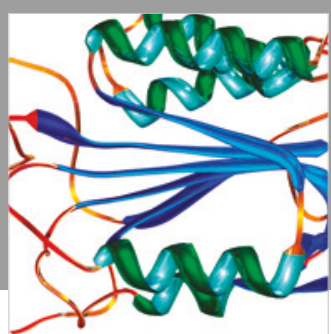

Disease Markers
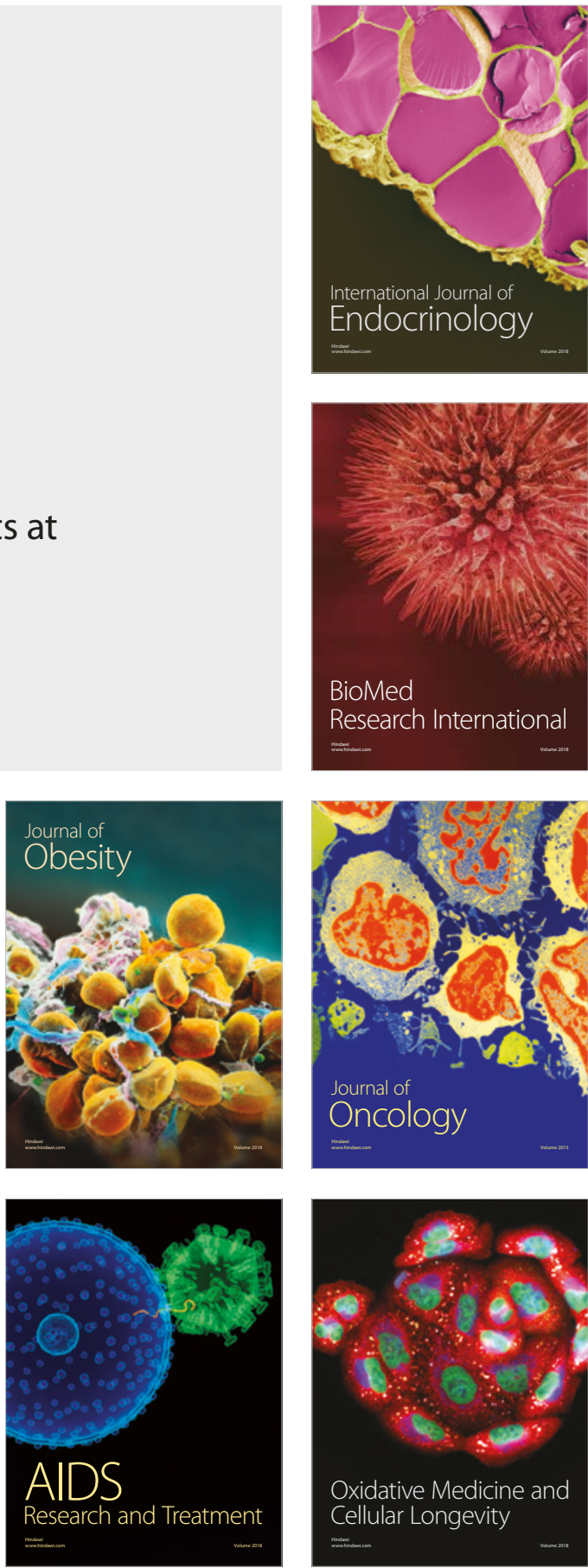